\section{Schonende Therapieoption: Mit hoher Energie gegen Prostata-Tumoren}

Eine schonende Therapieoption für Männer mit einem lokalisierten Prostatakarzinom im Frühstadium ist die Therapie mit hochintensivem fokussiertem Ultraschall. Es kommt kaum zu unerwünschten Wirkungen, etwa zu Harninkontinenz oder erektiler Dysfunktion. In einer kleinen britischen Studie ließen sich zudem nach einem Jahr bei 30 von 39 Patienten histologisch keine malignen Zellen mehr nachweisen.

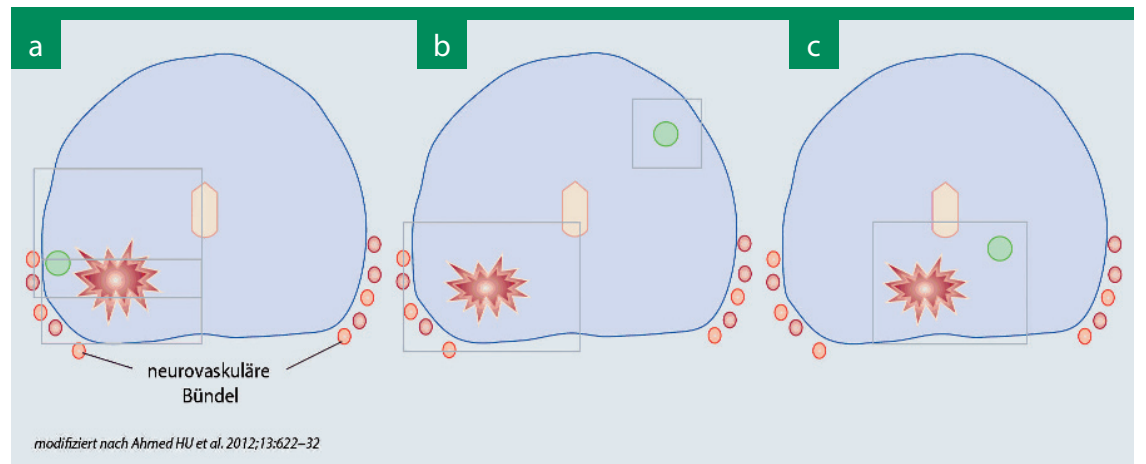

Abb.: Unilaterale „one-area“-Ablation (a), bilaterale „two-area”-Ablation mit Erhaltung von einem neurovaskulären Bündel (b), ,"one-area"-Ablation der Mittellinie mit Erhaltung beider neurovaskulärer Bündel (c). Große rote Gebiete repräsentieren dominante Tumorläsionen, kleine grüne Areale kleine niedriggradige sekundäre Läsionen. Graue Boxen stellen Ablationszonen auf dem hochintensiven fokussierten Ultraschallgerät dar.

$\mathrm{H}$ ochintensiver fokussierter Ultraschall (HIFU, Abb.) ist wie die Kryotherapie ein Mittelweg zwischen radikaler Prostatektomie und Radiotherapie, wie Peter Scardino aus New York, NY/USA, aus Anlass des 30. Deutschen Krebskongresses in Berlin 2012 es ausdrückte. HIFU wird derzeit eher in Europa, die Kryotherapie dagegen primär in den USA genutzt.

Bei dem Ultraschallverfahren, das wiederholt angewendet werden kann, wird ein gebündeltes Schallfeld über den Enddarm in die Prostata eingebracht. Im Brennpunkt des Schallfeldes entstehen Temperaturen von 95 bis $100^{\circ} \mathrm{C}$, es kommt zur Hitzekoagulation. Durch genaue computergesteuerte Definition des Behandlungsfeldes kann das gesamte Prostatavolumen, im Bedarfsfalle bis zur Organgrenze, behandelt werden. Eine HIFU unter lokaler und leichter Vollnarkose dauert zwischen zwei und drei Stunden. Spätestens nach einem Tag können die Patienten die Klinik wieder verlassen.

Britische Urologen haben das Verfahren jetzt bei über 40 Männern mit lokal begrenztem Prostatakarzinom im Frühsta-
Wie Hashim U. Ahmed und Kollegen vom University College in London berichteten, waren fast $90 \%$ der Männer auch ein Jahr nach der HIFU-Behandlung kontinent und gaben an, ein erfülltes Sexualleben haben zu können. Wenige Monate nach Beginn der Therapie begann der PSA-Wert zu sinken, und zwar von median $6,6 \mathrm{ng} / \mathrm{ml}$ zu Therapiebeginn auf 1,9 $\mathrm{ng} / \mathrm{ml}$ zwölf Monate später.

Für die histologische Auswertung standen Proben von 39 Männern zur Verfügung. Sechs Monate nach der Therapie ließen sich in den Biopsaten bei 30 von 39 Männern keine Tumorzellen mehr nachweisen. Nach Angaben von Ahmed und seinen Kollegen hatten alle Biopsate Zeichen einer Nekrose, einer Fibrose oder einer Riesenzellreaktion als Hinweis auf eine chronische Entzündung. Alle drei Parameter waren für sie ein Beleg dafür, dass sie mit HIFU das gewünschte Areal erreicht und zerstört hatten.

Auch in Deutschland wird die HIFUTherapie von vielen Kliniken angeboten und die Kosten werden im Allgemeinen im Rahmen eines stationären Aufenthaltes von den Krankenkassen übernommen. Die S3-Leitlinie zur Früherkennung Diagnostik und Therapie des Prostatakarzinoms enthält in der aktuellen Version von 2011 eine Bemerkung zu diesem Verfahren, in der es heißt, dass ein routinemäßiger Einsatz der HIFU zur Behandlung bei lokal begrenztem Prostatakarzinoms wegen fehlender Daten nicht gerechtfertigt sei.

Peter Leiner

Ahmed HU et al. Focal therapy for localised unifocal and multifocal prostate cancer: a prospective development study. Lancet Oncol. 2012;13(6):622-32.
Die Studie von Hashim U. Ahmed und Kollegen sollte aufgrund der kontinuierlichen Entwicklung von bildgebenden Verfahren und ablativen Techniken als eine frühe Arbeit betrachtet werden, kommentierten Matvey Tsivian, Michael R. Abern und Thomas J. Plascik vom Duke Cancer Institute in Durham, NC/USA. Denn nach ihren Ausführungen nutzten die Studienautoren nur den konventionellen Ultraschall, um die Ablation zu begleiten. Mit neueren Techniken wie Magnetresonanztomografie oder Ultraschallfusionsplattformen wäre eventuell eine noch präzisere Therapie möglich. Davon abgesehen begrüßten sie den gewebssparenden Effekt der fokalen Therapie, der Nebenwirkungen reduzieren und dadurch physiologische Funktionen erhalten könne, was sich womöglich positiv auf die Lebensqualität auswirken werde. Für künftige Untersuchungen forderten sie eine Standardisierung, die Definition von allgemeingültigen Behandlungschemata und Nomenklatur sowie optimierte Kriterien zur Auswahl der Patienten.

Tsivian M et al. Prostate cancer treatment unblended. Lancet Oncol. 2012;13:622-32. 